Textures and Microstructures, 1991, Vols 14-18, pp. 641-646 Reprints available from the publisher

Photocopying permitted by license only
(C) 1991 Gordon and Breach Science Publishers SA Printed in the United Kingdom

\title{
THE ORIENTATION CHARACTERISTICS OF DIFFERENT RECRYSTALLIZATION STAGES IN COPPER
}

\author{
K.SZTWIERTNIA*) and F.HAESSNER \\ Institut für Werkstoffe, Technische Universität Braunschweig, Germany
}

\section{INTRODUCTION}

The general features concerning the development of the recrystallization texture in a polycrystalline metal have been extracted from a large amount of experimental investigations. The main problem in this field is to quantify the relative significance of existing models or experimental data with respect to the global process of recrystallization texture formation. The reason for this must be seen in the fact that the phenomenon is a statistical one, taking place in a larger volume, whereas as a rule only information about a particular recrystallized state or about a locally closely confined region of the material is available.

In the present investigation the microstructure of the recrystallizer grains and their deformed surroundings, and local orientations of defined areas, are studied in the transmission electron microscope (TEM) for various stages of recrystallization of the same initial state. The results are then suitably statistically analysed and presented. The material parameters were chosen such that, in the deformed state, as broad a spectrum of orientations of the nucleation process as possible was obtained (high proportion of shear bands). The nucleation conditions so produced are particularly suitable for the investigation of the mechanisms of oriented growth.

\section{EXPERIMENTAL DETAILS}

Through special thermo-mechanical treatment soft copper samples (purity: $99,9998 \%$ ) were produced which showed a fairly random texture for a mean grain size of $100 \mu \mathrm{m}$. The material was $95 \%$ reversibly cold-rolled. The ratio $I_{d} / h \quad\left[l_{d}:\right.$ compressed length, $h$ : sample thickness before each rolling step] varied here between 1 to 5 . In this way homogeneous deformation conditions are ensured throughout the sample cross-section. By choosing different annealing periods (10 minutes and 1,5 hours respectively) for the rolled material at $140^{\circ} \mathrm{C}$, two primary recrystallization stages were generated which from calorimetric measurements correspond to a degree of recrystallization of about $10 \%$ and $50 \%$ respectively. The choice of just these recrystallization stages is based on earlier investigations of texture in rolled copper $/ 1 /$.

Using the cold-rolled material as well as the annealed samples, thin foils perpendicular to the normal direction ("N-Section") as well as to the transverse direction ("T-Section") were prepared. In the TEM, at an accelerating voltage of $120 \mathrm{kV}$, bright field images and Kikuchi-patterns (nanobeam electron diffraction) were made at defined sites. The orientations were calculated on-line from the Kikuchi-patterns /2,3/. The accuracy of the orientation determination is about $1^{\circ}$ in the reference system of the microscope. Since the determination of the sample coordinate system is rather more difficult the orientation specifications in the sample system are only accurate to within $5^{\circ}$.

*) On leave from the Instytut Podstaw Metalurgii, Kraków, Poland. 
The orientations were determined of both the recrystallized grains and the surrounding deformed areas. For both annealing states and both foil sections the following number of single orientations were studied:

10\% Series: $N$-Section 273, T-Section 69;

50\% Series: $\mathbf{N}$-Section 386, T-Section 122.

In addition the areas of the recrystallized grains were measured on the bright field images. For the $\mathrm{N}$-Sections the grains were divided into two groups: "small" and "large". Into the "small" group of grains belong all those that have an area smaller or equal to $10^{-2}$ of the area of the largest grain of a given series.

Using the orientation data for the individual sites, two types of distribution function were calculated:

a) The Orientation Distribution Function (ODF) of the recrystallized grains and the ODF of the neighbouring fragments of the deformed matrix.

b) The Misorientation Distribution Function (MDF) between the recrystallized grains and the fragments of the deformed matrix.

Both types of function were calculated from single orientations or misorientations by the method of Fourier series expansion of generalized spherical harmonics $/ 4 /$. The ODF's are shown in the Euler space $\varphi_{1}(F \mid 1), \Phi(F I), \varphi_{2}(F I 2)$ in cross-sections $\varphi_{2}=$ const. The MDF's are presented in the axis-angle space in the basic domain of the smallest rotation angele $\omega_{d}$ in cross - sections $\omega=$ const.

\section{RESULTS AND CONCLUSIONS}

The microstructure of rolled copper consists of grains elongated in rolling direction lying parallel to the sheet plane. Shear bands are fairly often observed. The ODF's calculated from single orientations for the deformed state, as well as those for the fragments of the deformed matrix for the two intermediate recrystallization stages, do not essentially differ from each other. They can be described by means of two main components $\sim\{123\}<634>$ and $\sim\{112\}<111>$, typical of the copper type rolling texture, and of two minor components $\{011\}<211>$ and $\{011\}<100>$.

At the initial stage of recrystallization $(\sim 10 \%$ recrystallized) the ODF of the new grains in the $\mathrm{N}$-section is fairly complicated and moreover different for small and large grains (Fig.1a,b). The orientations of the small grains are scattered to a large extend around the deformation components $\{123\}<634>,\{112\}<111>$ and $\{011\}<211>$. For the large grains, on the other hand, the orientations scatter mainly between the positions $\{122\}<212>+\{123\}<634>$ (the disorientation angle $\omega_{\mathrm{d}}$ between $\{122\}<212>$ and $\{123\}<634\}$ is only $11,7^{\circ}$ ) and $\{011\}<111>$. The $\{001\}<100>$ component occurs but is only relatively weakly defined. The grains of the $T$-section yield a similar result.

The misorientation distributions between the recrystallized grains and the surrounding matrix in no case exhibit a tendency to a random distribution. The orientation parameters of the position of the maxima in the MDF's often can be approximated by coincidence 
orientation relationships. This is shown by the MDF's represented in Figs. $3 a$ and $b$ for the $\mathrm{N}$-section as an example. Here for the small grains the $\left.60^{\circ}<111\right\rangle(\Sigma=3)$ relationship occurs preferentially, as well as misorientations with small angles of rotation, whereas in the case of the large grains it is the $\left.-35^{\circ}<112\right\rangle \quad(\Sigma=35 \mathrm{a})$ relationship. The situation in the $T$-section shows that, besides small angles of rotation, there are rotations in the ranges $35-60^{\circ}<111>$ or $15^{\circ}-30^{\circ}<110>$.

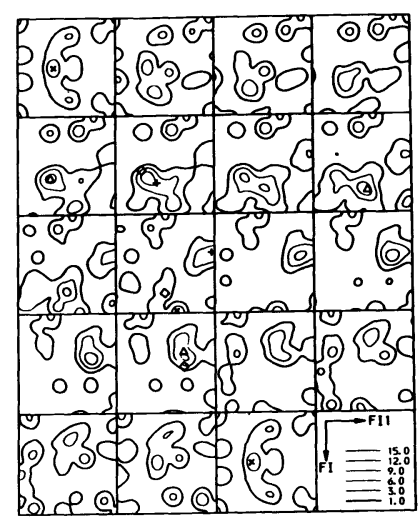

(a)

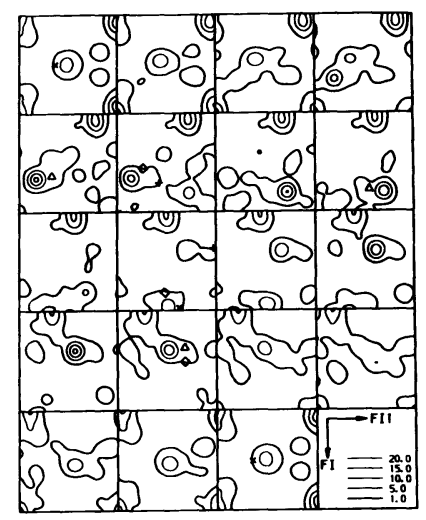

(c)

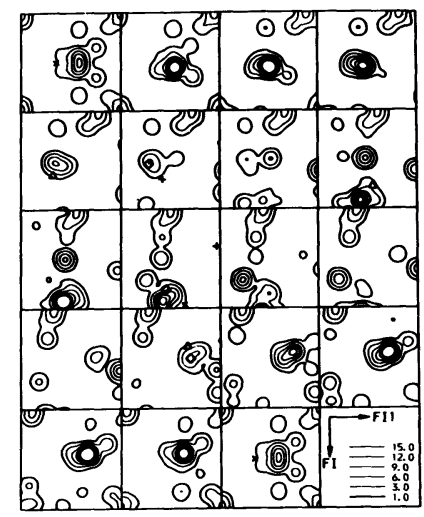

(b)

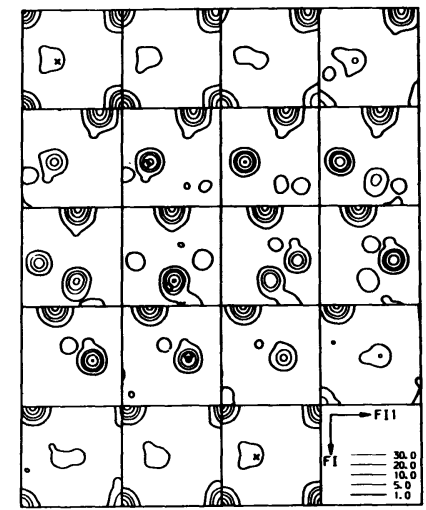

(d)

$X\{011\}<211>+\{112\}<111>\Delta\{123\}<634>\quad \diamond\{122\}<212>$

Fig.1: ODFs of recrystallized grains in $95 \%$ rolled copper, annealed at $140^{\circ} \mathrm{C}$ for different times:

a) $10 \mathrm{~min}$ ( $\mathrm{N}$-section; 103 "small" grains).

b) $10 \mathrm{~min}$ ( $\mathrm{N}$-section; 16 "large" grains).

c) $1,5 \mathrm{~h} \quad(\mathrm{~N}$-section; 65 "large" grains).

d) $1,5 \mathrm{~h}$ ( $\mathrm{T}$-section; 82 grains).

At the intermediate stage of recrystallization ( $\sim 50 \%$ recrystallized) the ODF of the new large grains shows great similarity (particularly in the $T$-section) with the primary recrystallization texture of pure copper, which can be described by two components $\{001\}<100>$ and $\{122\}<212>$ (Fig.1C,d). The orientations of the new small grains lie 
close to the components of the deformations texture or close to the cube position.

Quite frequently small grains with orientation $\sim\{001\}<100>$ were found in contact with large primary grains of $\sim\{122\}<212>$ orientation. These small grains were twins of the large grains.

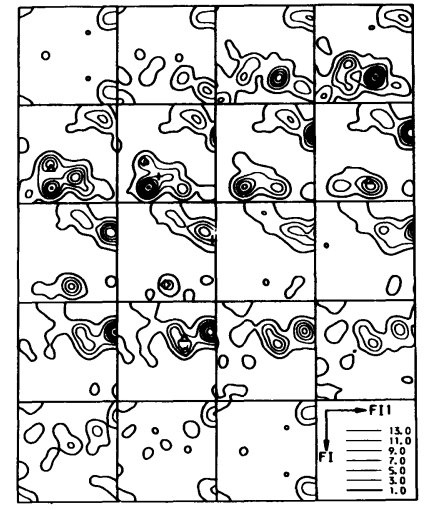

(a)

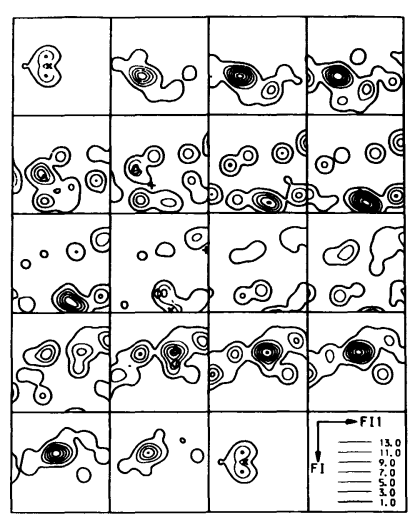

(b)

$$
\times\{011\}<211>+\{112\}<111>\Delta\{123\}<634>\diamond\{122\}<212>
$$

Fig.2: ODFs of fragments of the deformed matrix lying close to recrystallized grains $(95 \%$ rolled copper, annealed $1,5 \mathrm{~h}$ at $140^{\circ} \mathrm{C}$ ).

a) neighbourhood of $\{001\}<100>$ oriented grains (57 orientations).

b) neighbourhood of $\{122\}<212>$ oriented grains (30 orientations).

The misorientation distribution between the large recrystallized grains and the surrounding matrix shows a fairly strong maximum at $40^{\circ}\langle 111\rangle$, but also misorientations at $60^{\circ}<111>, 40^{\circ}<101>, 50^{\circ}<101>, 30^{\circ}<111>, 25^{\circ}<101>, 40^{\circ}<113>$ and $30^{\circ}<101>$. They all correspond to coincidence orientation relationships with $\Sigma$ equal to $7,3,9,11,13 b, 19 a, 23$ and $27 a$ respectively (Fig.3d). For the small grains the relationships $\left.\left.60^{\circ}<111\right\rangle, 40^{\circ}<101\right\rangle$ and misorientations with small angles of rotation occur. In the interval of $\omega_{\mathrm{d}} 20-60^{\circ}$ possible axes of rotation scatter strongly around $<111>$. The situation in the $T$-section shows, besides strong maxima at $60^{\circ}<111>$ and $50^{\circ}<210>$ $(\Sigma=15)$ rotations in the ranges $10-20^{\circ}$ and $30-40^{\circ}<101>, 40^{\circ}<111>, 40-45^{\circ}$ between $<211>$ and $<221>, 35-55^{\circ}<331>$.

The orientations of the deformed surroundings were selected separately for the recrystallized grains of type $\{001\}<100>$ and $\{122\}<212>$. Figs.2a,b show the ODFs for these two cases: For the $\{001\}<100>$ grains orientations are found in the surroundings close to $\{123\}(634)$ and $\{112\}<111>$; for the $\{122\}<212>$ grains the neighbouring orientations lie close to $\{123\}<634>$ or to $\{122\}<212>$. The $\{001\}<100>$ oriented new grains in the sample are locally very inhomogeneously distributed. Besides foils that do not contain a single one of these grains, there are others that are particularly rich in them. By way of contrast, the local distribution of the $\{122\}<212>$ grains is very uniform. 


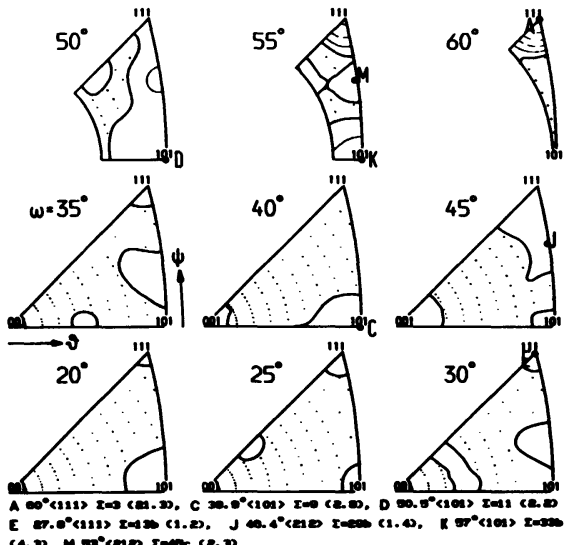

(a)

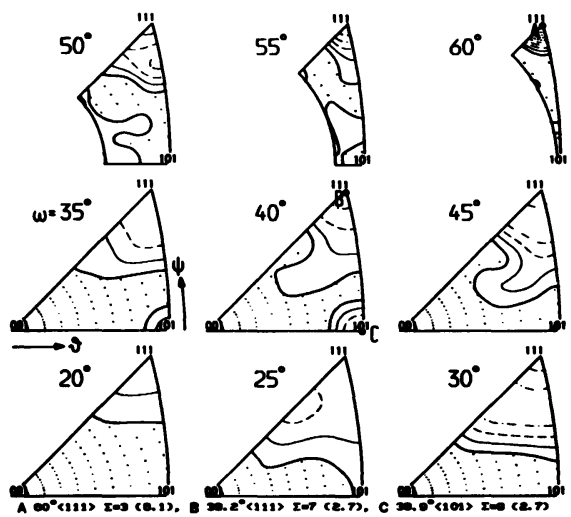

(c)
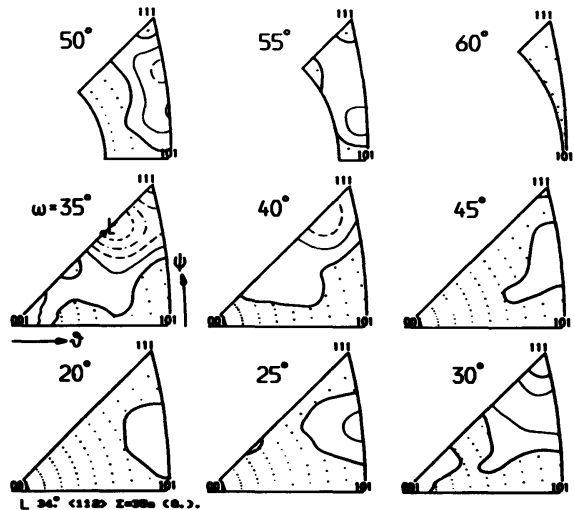

(b)

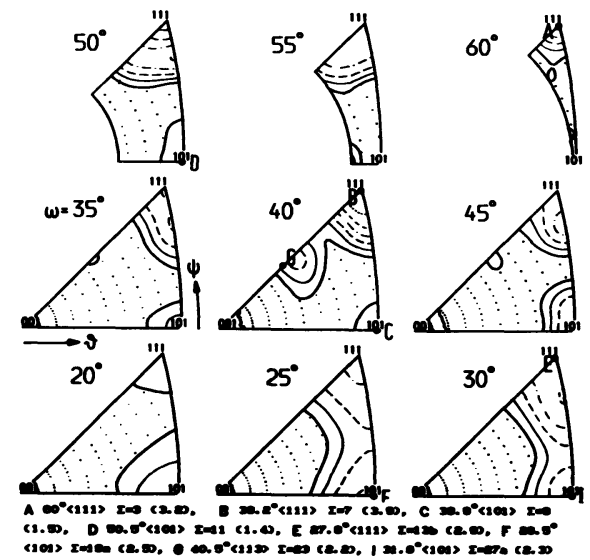

(d)

Fig.3: MDFs of misorientations between the recrystallized grains and the surrounding matrix in $95 \%$ rolled copper, annealed for different times:

a) $10 \mathrm{~min}$, set of $\mathbf{1 5 0}$ misorientations between "small" grains and the matrix, contour lines 1., 3., 6., 10., 15. .

b) $10 \mathrm{~min}$, set of 44 misorientations between "large" grains and the matrix, contour lines 1., 2., 3., ... .

c) 1,5 $\mathrm{h}$, set of 118 misorientations between "small" grains and the matrix, contour lines 1., $1.5,2 ., \ldots$.

d) 1,5 h, set of 158 misorientations between "large" grains and the matrix, contour lines 1., $1.5,2 ., \ldots$.

(Only the $\mathrm{N}$-sections. Regions under the level 1. are dotted. Positions of CSL orientation relationships marked by: $A, B, \ldots$, angle-axis description, value of $\Sigma$ and the value of the nearest maxima in the MDF). 
The different ODF's and MDF's for small and large grains, and for the two recrystallization stages, indicate that the oriented growth of the nuclei has considerable effect on the generation of the primary recrystallization texture. We shall look at this more closely for two very remarkable results:

(i) The ODF of the small grains at the start of the process (Fig.1) yields information on the distribution of the nuclei. Their orientations scatter around the components of the deformation texture or are related to it by twinning. It is remarkable that, of these nuclei in this stage only the $\sim\{123\}<634>$ and $\sim\{122\}<212>$ oriented ones grow, though not for instance the $\{112\}<111>$ oriented nuclei (Fig.1b). The reason for this may be that a $\sim\{112\}<111>$ nucleus either shows a $\sim 60^{\circ}<111>$ orientation relation with the deformed surroundings (for complementary components), or has a very small misorientation. In both cases the mobility of the boundary is very low. On the other hand, a $\sim\{123\}<634>$ nucleus has a $\sim 40^{\circ}<111>$, $\sim 40^{\circ}<221>$ or $\sim 50^{\circ}<221>$ orientation relation (for complementary components) or a very small misorientation. At least the mobility of a boundary between $40^{\circ}<111>$ misoriented regions is not low. This means that the results described can without difficulty be attributed to oriented growth.

(ii) The recrystallization component $\sim\{122\}<212>$ is usually interpreted as a twin of the first generation of the cube component. The relationships here, certainly for some of the $\sim\{122\}<212>$ components, are the opposite $/ 5 /:$ The $\sim\{122\}<212>$ component is represented much more strongly at the start of recrystallization than the cube component (Fig.1b). During further annealing, based on our morphological observations, it would appear that a considerable number of $\sim\{001\}<100>$ oriented small grains are created by twinning in or near the $\sim\{122\}<212>$ oriented regions. The cube orientation can grow quickly as a compromise position to the components of the deformation texture. In this way it determines the recrystallization texture at the advanced stages of the process (Fig.1d). In accordance with this interpretation are the strong $40^{\circ}<111>$ maximum of the MDF (Fig.3d) and the different ODF's for the environment of the $\{001\}<100>$ and $\{122\}<212>$ grains (Fig.2a,b).

\section{ACKNOWLEDGEMENT}

This work has been supported by the Deutsche Forschungsgemeinschaft.

\section{REFERENCES}

1. K.Sztwiertnia and F.Haeßner, in "Recrystallization ' 90 " p.535, Ed.T.Chandra, The Minerals, Metals \& Materials Society, 1990.

2. P.Heilmann, W.A.T.Clark and D.A.Rigney, Ultramicroscopy 9, 365 (1982).

3. H.Weiland and R.Schwarzer, in "Experimental Techniques of Texture Analysis" p.301, (H.J.Bunge, DGM Informationsgesellschaft, Oberursel, 1986).

4. H.J.Bunge, Texture Analysis in Materials Science, Butterworths, London 1982.

5. B.J.Duggan, M.Sindel, G.D.Kölhoff and K.Lücke, Acta metall.mater. 38, 103 (1990). 